\title{
Dynamic MRI Sensitized to Cerebral Blood Oxygenation and Flow During Sustained Activation of Human Visual Cortex
}

\author{
Gunnar Krüger, Andreas Kleinschmidt, Jens Frahm
}

\begin{abstract}
Changes in cerebral blood oxygenation and flow during prolonged activation of human visual cortex (6-min video projection) were monitored using high-resolution $T_{2}{ }^{*}$ - and $T_{1}$-weighted gradient-echo MRI in identical sessions. Oxygenation-sensitive recordings displayed an initial signal increase (oxygenation "overshoot"), a subsequent signal decrease extending over 4-5 min (relative deoxygenation), and a signal drop after the end of stimulation that mirrored the initial response (oxygenation "undershoot"). Flow-sensitive MRI demonstrated that the inflow effect remained elevated during the entire period of stimulation. The observation of gradually decreasing cerebral blood oxygenation, despite persisting elevation of blood flow, may be understood to be an accumulation of deoxyhemoglobin due to the progressive up-regulation of oxidative phosphorylation. The present findings support a concept in which transitions between functional states lead to an uncoupling of perfusion (oxygen delivery) from oxidative metabolism (oxygen consumption) whereas steady-state activity achieves their recoupling.
\end{abstract}

Key Words: brain pertusion; cerebral blood flow; cerebral blood oxygenation; brain; metabolism.

\section{INTRODUCTION}

In a recent study involving the use of combined proton MRS and gradient-echo MRI sensitized to changes in cerebral blood oxygenation (CBO), we provided evidence for a merely initial prevalence of previously suggested nonoxidative glycolysis in human cortex $(1,2)$ in response to a major increase in neural activity (3). During such transitions of neuronal activity, blood flow and glucose uptake increase and show a physiological "uncoupling" from largely unaffected oxygen consumption. In the aforementioned study, we detailed its temporal evolution and thereby reconciled this observation with the classic understanding of the general coupling of perfusion and oxidative metabolism (4). Within a 6-min period of visual stimulation, CBO-sensitive MRS and

\footnotetext{
MRM 35:797-800 (1986)

From the Biomedizinische NMR Forschungs GmbH am Max-Planck-Institut für biophysikalische Chemie Am Fassberg 11, D-37077 Göttingen, Germany.

Address correspondence to: Jens Frahm, Ph.D., Biomedizinische NMR Forschungs GmbH am Max-Planck-Institut für biophysikalische Chemie Am Fassberg 11, D-37077 Göttingen, Germany.

Received September 7. 1995; revised December 4, 1995; accepted February $1,1996$.

0740-3194/96 $\$ 3.00$

Copyright $\odot 1996$ by Williams \& Wilkins

All rights of reproduction in any form reserved.
}

MRI independently revealed a signal intensity decrease to prestimulus baseline, indicating that steady-state conditions are accompanied by "recoupling." However, the assignment of these signal decreases to an up-regulation of oxidative metabolism, and concomitantly enhanced oxygen consumption assumes that blood flow remains constantly elevated throughout the experiment. This study specifically addresses this issue by parallel investigations of stimulus-related changes in both regional CBO and blood flow using dynamic gradient-echo MRI with respective contrasts.

\section{METHODS}

Studies of 10 healthy human subjects (age 24-52 years, mean 29.5 years) were conducted at 2.0 T (Siemens Mag. netom SP4000, Erlangen, Germany) using the standard imaging headcoil. Visual stimulation involved a 6-min video presentation (medical education, color, no sound) with use of a specially designed MRI projection setup covering $40^{\circ} \times 30^{\circ}$ of the visual field (Schäfter and Kirchhoff, Hamburg, Germany). The material contained demonstrations of diagnostic equipment and procedures. No part of the video was presented twice, and subjects were free to move their eyes. Attention was ensured by informing subjects that they would be interviewed on the content of the clips after the session. Total protocol duration was 12 min. Additionally, subjects underwent a repetitive protocol comprising six cycles with $18 \mathrm{~s}$ of stimulation (same video) and $36 \mathrm{~s}$ of darkness each. These measurements were immediately evaluated and served to verify sufficient visual responsiveness in the sections chosen on anatomical grounds.

Dynamic CBO-sensitive MRI (RF-spoiled FLASH, $T R / T E=62.5 / 30 \mathrm{~ms}$, flip angle $10^{\circ}$ ) was performed using spin-density weighting with $T_{2}{ }^{*}$ sensitivity (5). Flowsensitive recordings were obtained by emphasizing inflow phenomena with strong $T_{1}$ weighting but $T_{2}$ * insensitivity (RF-spoiled FLASH, TR/TE $=62.5 / 7.5 \mathrm{~ms}$, flip angle $50^{\circ}$ ). The 12 -min span of the sustained stimulation protocol comprised of 60 sequential images (two averages of 6-s acquisitions). Sections $(4 \mathrm{~mm})$ were individually aligned to cover major parts of calcarine cortex with two to three repetitions of CBO-sensitive and flow-sensitive recordings each.

Activation was determined by correlating signal intensity time courses of each pixel with a reference waveform (6). For multiple repetitions of short stimuli, the reference was a box-car waveform shifted by one image ( $6 \mathrm{~s}$ ) 


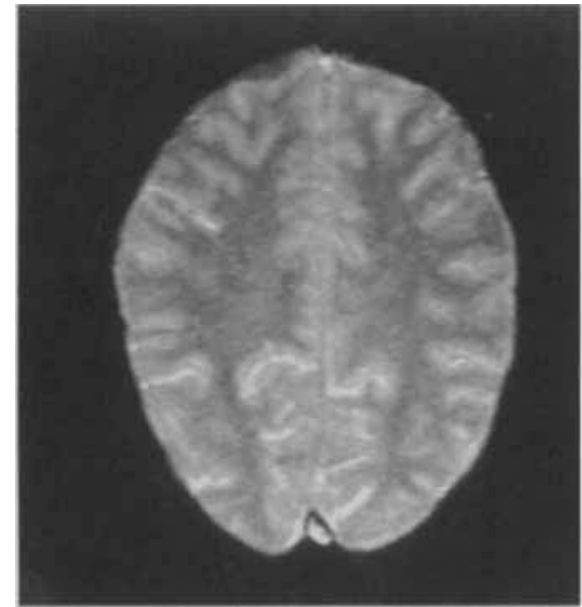

a

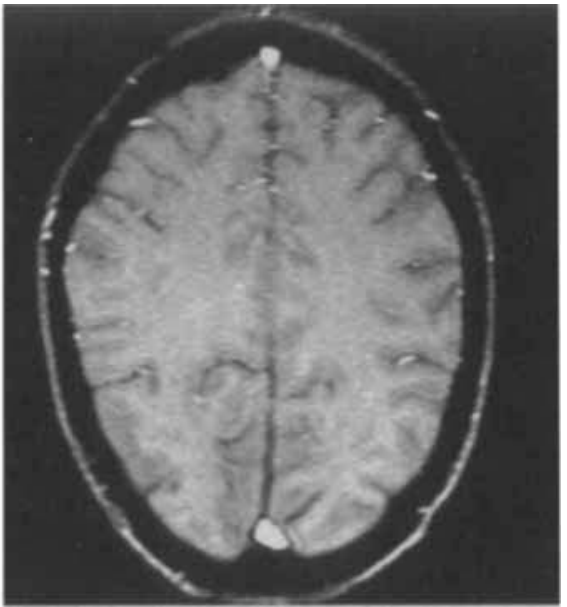

b relative to the protocol. For sustained stimulation, specific response functions for $\mathrm{CBO}$ - and flow-sensitive measurements were obtained by averaging signal-intensity time courses across subjects in areas identified by a correlation with the stimulus protocol. Separate "overshoot" and "undershoot" activation maps were obtained by correlating signal intensities from either the first or last $6 \mathrm{~min}$ of the 12-min MRI data set with respective parts of the mean CBO-sensitive response function. Quantitative maps of correlation coefficients were analyzed, thresholded, and color-coded as described previously (7). No temporal or spatial filters were applied. Regional time courses of signal-intensity changes were derived from all pixels showing significant alterations, i.e., with a $P \leq 0.0001$ corresponding to about three false-positive pixels admitted per dynamic MRI series.

\section{RESULTS AND DISCUSSION}

Figure 1 shows typical high-resolution $T_{2}{ }^{*}$ - and $T_{1}$ weighted single-slice gradient-echo images used to monitor changes dynamically in $\mathrm{CBO}$ and flow, respectively. Pertinent time courses from activated regions are depicted in Fig. 2 for $\mathrm{CBO}$ - and flow-sensitive recordings and demonstrate the grand average response observed in the study group (mean values $\pm S D$ ). In general agreement with previous studies of sustained flickerlight stimulation using both proton MRS and gradient-echo MRI (3), the CBO data (Fig. 2a) revealed a rapid signal increase or hyperoxygenation associated with stimulus onset followed by a signal decrease or relative deoxygenation extending over 4-5 min. Signal intensities toward the end of stimulation remained slightly elevated in comparison with prestimulus baseline (statistically not significant). After the end of stimulation, a pronounced and rapid signal drop or deoxygenation occurred that mirrored the initial hyperoxygenation in magnitude and recovery time.

In contrast, the flow-sensitive data (Fig. $2 \mathrm{~b}$ ) revealed a constant elevation of signal intensity due to inflow enhancement during sustained stimulation. Poststimulus intensity showed no undershoot phenomenon. Although there is not direct access to neuronal activity in func-
FIG. 1. Rapid low-flip-angle gradient-echo MRI covering the calcarine cortex of a healthy volunteer. (a) CBO-sensitive acquisition using spin-density weighting with $T_{2}$ " sensitivity (RF-spoiled FLASH, TR = $62.5 \mathrm{~ms}, T E=30 \mathrm{~ms}$, flip angle $10^{\circ}$, in plane resolution $0.78 \times 1.56 \mathrm{~mm}^{2}$ interpolated to $0.78 \times 0.78 \mathrm{~mm}^{2}$, slice thickness $4 \mathrm{~mm}$ ). (b) Flow-sensitive acquisition using strong $T_{1}$ weighting without $T_{2}^{*}$ sensitivity (TE $=7.5 \mathrm{~ms}$, flip angle $50^{\circ}$, other parameters as indicated for a).
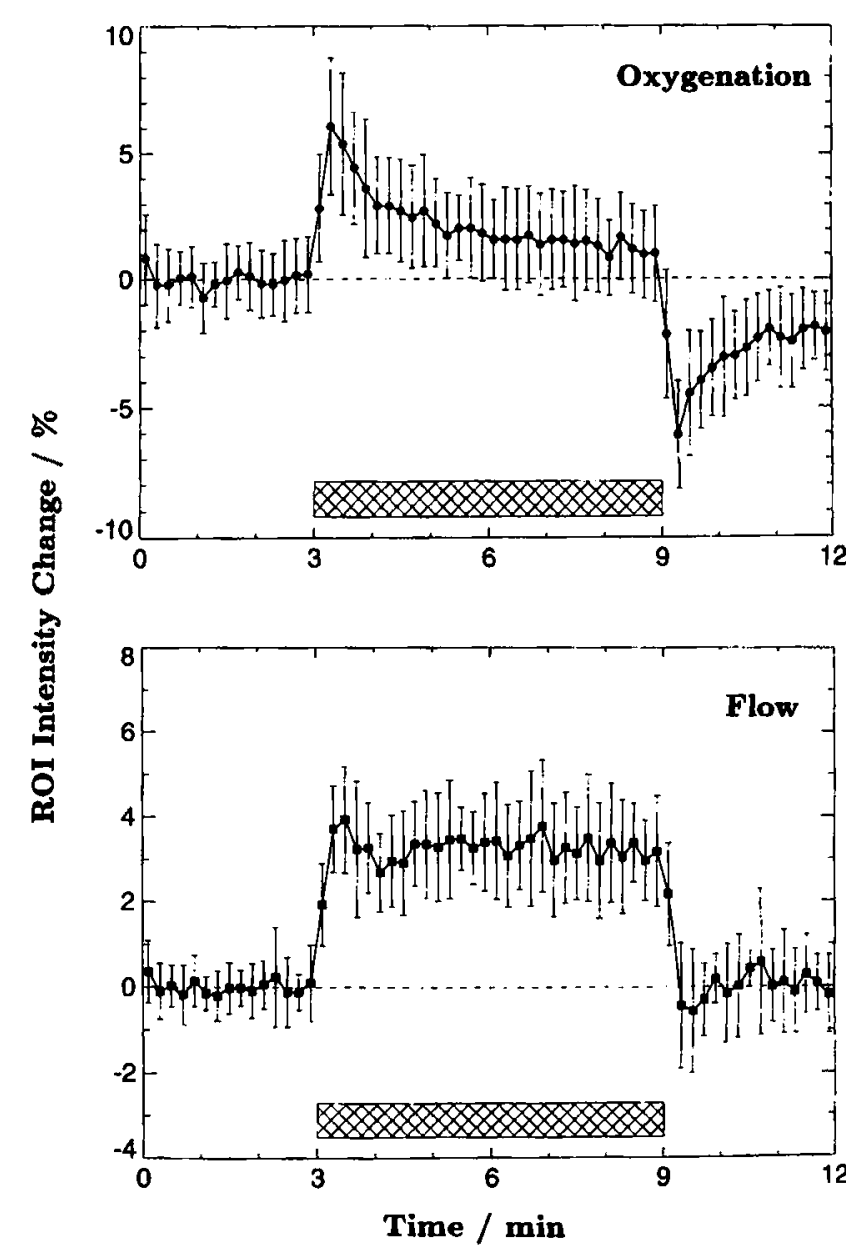

FIG. 2. Normalized regional time courses of MRI signal intensities (12-s temporal resolution) sensitized to changes in (a) CBO and (b) blood flow during a 12-min protocol comprising 6 min of visual stimulation (cross-hatched bar). The values represent mean $\pm S D$ for all subjects $(n=10)$ in areas exhibiting stimulus-related signal alterations. As demonstrated by the small fluctuations of the mean response, the error bars mostly reflect interindividual differences rather than physiological noise during the individual recordings. 

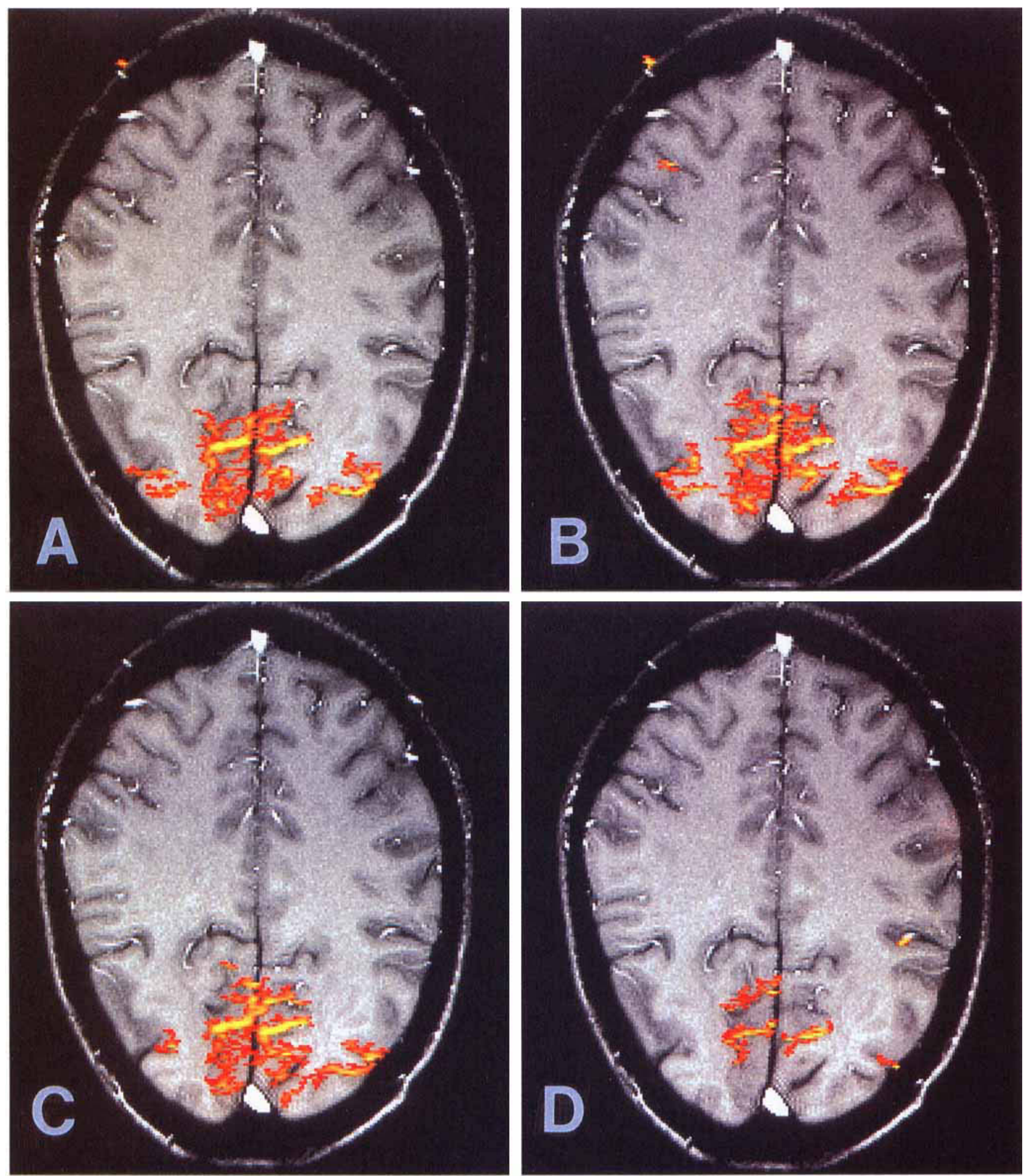

FIG. 3. Color-coded activation maps superimposed onto an anatomical reference image for the same subject as in Fig. 1. The maps demonstrate spatial congruence of brain regions that exhibit (a) an initial oxygenation overshoot after stimulus onset, (b) a terminal oxygenation undershoot after ending stimulation, and (c) MRI responses in correlation with a repetitive protocol comprising six cycles of $18 \mathrm{~s}$ of stimulation and $36 \mathrm{~s}$ of darkness each. (d) Correlation map of flow-sensitive responses during 6 min of stimulation.

tional neuroimaging, the persistence of flow-sensitive responses as well as the marked poststimulus undershoot of CBO-sensitive responses argue against contributions from diminished attention or habituation. Another potential source of CBO signal reduction could be a slow increase in cerebral blood volume by (presumably venous) vasodilation, but it seems unlikely that this should exclusively affect CBO- but not flow-sensitive recordings. The reason for a discrepancy of the CBO-sensitive data with a preliminary report using echo-planar imaging 
at a 30-fold lower spatial resolution (8) remains an unresolved issue. Putative explanations include technical properties that result in different MRI contrasts and image sensitivities as well as stimulus properties that elicit responses of different strengths and/or involve other subsystems and thus may cause variable demands for oxidative metabolism.

The present findings support a model in which not only hemodynamic but also oxidative metabolic adjustments occur in response to functional challenge. As shown in Fig. 3, differences in their temporal evolution are responsible for both the initial hyperoxygenation (Fig. 3a) and the deoxygenation after end of stimulation (Fig. 3b). Pertinent signal changes are equivalent in strength and observed in identical regions. Thus, all brain regions that exhibit an activation-related oxygenation overshoot also generate an undershoot with deactivation. Moreover, activated areas in such maps are congruent with regions defined by conventional "mapping" with several brief cycles of stimulation (Fig. 3c), whereas detectable alterations of flow were confined to more restricted areas most likely encompassing larger veins (Fig. 3d).

In synopsis with the metabolic data presented previously (3), the temporal evolution of focal brain activation may be characterized by the following sequence of events. After the first few seconds of hemodynamic latency, the rise in perfusion, i.e., oxygen and glucose delivery, remains without a corresponding increase in oxygen consumption. The resulting postcapillary oxygenation overshoot provides CBO-sensitive MRI contrast. In the first 2-3 min, increased glucose consumption with transient lactate accumulation indicates enhanced nonoxidative glycolysis. This initial phase is characterized by the uncoupling of perfusion and oxidative metabolism. After 4-5 min, the continuous elevation of blood flow and glucose consumption is progressively matched by an up-regulation of oxidative metabolism that recouples oxygen consumption in a new steady state with CBO reduced close to or identical with baseline values. Finally, terminating activation causes a rapid normalization in blood flow, i.e., oxygen and glucose delivery, that yields a transient oxygenation undershoot due to the persistence and gradual attenuation of elevated oxygen consumption. Analogous to the onset of activation, the physiological processes linked to deactivation may be considered as a transient "negative" uncoupling of perfusion and oxidative metabolism. Consequently, the level of blood flow seems to reflect the degree of neuronal activity in a functional state, whereas $\mathrm{CBO}$ is mainly sensitive to activation as the switching between functional states.

\section{ACKNOWLEDGMENTS}

The authors thank the Gesellschaft für wissenschaftliche Datenverarbeitung Göttingen for data analysis and figure preparation.

\section{REFERENCES}

1. P. T. Fox, M. E. Raichle, Focal physiological uncoupling of cerebral blood flow and oxidative metabolism during somatosensory stimulation in human subjects. Proc. Natl. Acad. Sci. USA 83, 1140-1144 (1986).

2. P. T. Fox, M. E. Raichle, M. A. Mintun, C. Dence, Nonoxidative glucose consumption during focal physiologic neural activity. Science 241, 462-464 (1988).

3. J. Frahm, G. Krüger, K. D. Merboldt, A. Kleinschmidt, Dynamic uncoupling and recoupling of perfusion and oxidative metabolism during focal brain activation in man. Magn. Reson. Med. 35, 143-148 (1996).

4. D. D. Clarke, L. Sokoloff, Circulation and energy metabolism of the brain in "Basic Neurochemistry" (G. J. Siegel, B. W. Agranoff, R. W. Albers, P. B. Molinoff, Eds.), pp. 645-680, Raven, New York, 1994.

5. J. Frahm, K. D. Merboldt, W. Hänicke, A. Kleinschmidt, H. Boecker, Brain or vein-oxygenation or flow? On signal physiology in functional MRI of human brain activation. NMR Biomed. 7, 45-53 (1994).

6. P. A. Bandettini, A. Jesmanowicz, E. C. Wong, J. S. Hyde, Processing strategies for time-course data sets in functional MRI of the human brain. Magn. Reson. Med. 30, 161-173 (1993).

7. A. Kleinschmidt, M. Requardt, K. D. Merboldt, J. Frahm, On the use of temporal correlation coefficients for magnetic resonance mapping of functional brain activation. Individualized thresholds and spatial response delineation. Intern. J. Imaging Sys. Technol. 6, 238-244 (1995).

8. P. A. Bandettini, T. L. Davis, K. K. Kwong, P. T. Fox, A. Jiang, J. R. Baker, J. W. Belliveau, R. M. Weisskoff, B. R. Rosen, FMRI and PET demonstrate sustained blood oxygenation and flow enhancement during extended visual stimulation durations, in "Proc. SMR, 3rd Annual Meeting, Nice, 1995," p. 453. 